\title{
PRECOMPACT APARTNESS SPACES
}

\author{
DOUGLAS S. BRIDGES
}

Department of Mathematics \& Statistics, University of Canterbury, Private Bag 4800, Christchurch 8140, New Zealand

e-mail address: d.bridges@math.canterbury.ac.nz

\begin{abstract}
We present a notion of precompactness, and study some of its properties, in the context of apartness spaces whose apartness structure is not necessarily induced by any uniform one. The presentation lies entirely with a Bishop-style constructive framework, and is a contribution to the ongoing development of the constructive theories of apartness and uniformity.
\end{abstract}

\section{INTRODUCTION}

The apparent difficulty of producing a decent notion of compactness for the constructive 1 theory of pre-apartness spaces has provoked several responses [6, 10, 14]. In this paper we offer a partial response - precompactness - without claiming, or even suggesting, that it may be the "right" one: we prefer to leave our offer on the table, to be considered further and then judged alongside those of other mathematicians who have considered the compactness problem.

Let $X$ be an inhabited set with an inequality relation $\neq$, and let $\bowtie$ be a relation between subsets of $X$ that satisfies certain axioms to be stated shortly. We define three types of complement for a subset $S$ of $X$ :

$$
\begin{aligned}
\neg S & \equiv\{x \in X: x \notin S\}, \\
\sim S & \equiv\left\{x \in X: \forall_{s \in S}(x \neq s)\right\}, \\
-S & \equiv\{x \in X:\{x\} \bowtie S\} .
\end{aligned}
$$

For $x \in X$, we normally write $x \bowtie S$ rather than $\{x\} \bowtie S$. We call $\bowtie$ a pre-apartness on $X$ if it satisfies these four axioms:

(B1) $X \bowtie \varnothing$

(B2) $-A \subset \sim A$

1998 ACM Subject Classification: G.m.

2010 Mathematics Subject Classification: 03F60, 54E05, 54E15.

Key words and phrases: constructive, apartness, uniform, precompact.

${ }^{1}$ For us, constructive mathematics means mathematics in the style of Errett Bishop: that is, mathematics developed with intuitionistic logic and some foundational system such as the constructive set theory in [1, 2] or the type theory in $[12$. We shall assume that the reader is familiar with, or has access to, the fundamentals of constructive analysis, as found in [4, 5, 8] (see also [3, 15]) 
(B3) $\left(\left(A_{1} \cup A_{2}\right) \bowtie\left(B_{1} \cup B_{2}\right)\right) \Leftrightarrow \forall_{i, j \in\{1,2\}} A_{i} \bowtie B_{j}$

(B4) $-A \subset \sim B \Rightarrow-A \subset-B$.

Taken with the relation $\bowtie$, the set $X$ then becomes a pre-apartness space. If, in addition, $\bowtie$ satisfies 2

(B5) $x \in-A \Rightarrow \exists_{S \subset X}(x \in-S \wedge X=-A \cup S)$,

then it is called an apartness on $X$, and $X$ becomes an apartness space.

There is a natural topology associated with a pre-apartness space: the apartness topology, in which the apartness complements form a base of open sets. It is this topology that we refer to when we consider notions like open, closed, and dense below.

Perhaps the most important example of an apartness space is given by a uniform space: that is, an inhabited set $X$ with a uniform structure $\mathcal{U} 3$ In this case, the apartness is defined, for subsets $S, T$ of $X$, by

$$
S \bowtie T \Leftrightarrow \exists_{U \in \mathcal{U}}(S \times T \subset \sim U) .
$$

The apartness topology corresponding to this apartness relation coincides with the usual uniform topology on $X$. Note that, in contrast to the classical situation, for us a uniform space is Hausdorff, by definition. Moreover, the inequality relation defined on $X$ by

$$
x \neq y \Leftrightarrow \exists_{U \in \mathcal{U}}((x, y) \notin U)
$$

is tight:

$$
\neg(x \neq y) \Rightarrow x=y .
$$

Since the uniform/apartness topology is Hausdorff and the inequality is tight, convergent nets in a uniform space have unique limits (9], Corollary 2.4.8).

A particular case occurs when $(X, \rho)$ is a metric space, when the uniform structure $\mathcal{U}$ has a base comprising sets of the form

$$
\left\{\left(x, x^{\prime}\right) \in X \times X: \rho\left(x, x^{\prime}\right)<\varepsilon\right\},
$$

where $\varepsilon>0$; then

$$
S \bowtie T \Leftrightarrow \exists_{\varepsilon>0} \forall_{s \in S} \forall_{t \in T}(\rho(s, t) \geq \varepsilon) .
$$

A uniform space $(X, \mathcal{U})$ is said to be totally bounded if for each $U \in \mathcal{U}$ there exist finitely many points $x_{1}, \ldots, x_{n}$ of $X$ such that

$$
X=\bigcup_{i=1}^{n}\{y \in X:(x, y) \in U\} .
$$

In the case of a metric space $X$, this reduces to the familiar elementary property of total boundedness. The closure of a totally bounded subset of a uniform space is totally bounded (9], Proposition 3.3.5). Moreover, as is straightforward to show, a dense subspace of a totally bounded uniform space is totally bounded. iff

A mapping $f: X \rightarrow Y$ between pre-apartness spaces is said to be strongly continuous

$$
\forall A, B \subset X(f(A) \bowtie f(B) \Rightarrow A \bowtie B) .
$$

\footnotetext{
${ }^{2}$ (B4) can actually, and easily, be derived from (B1)-(B3) and (B5); see page 69 of 9 .

${ }^{3}$ We omit the detailed definition of uniform structure, referring the reader to Section 3.2 of 9 for more information about apartness and uniform spaces.

${ }^{4}$ Strongly continuous mappings are the morphisms in the category of pre-apartness spaces.
} 
If $(X, \mathcal{U})$ and $(Y, \mathcal{V})$ are uniform spaces, $f: X \rightarrow Y$ is strongly continuous, and $f(X)$ is totally bounded, then $f$ is uniformly continuous in the sense that

$$
\forall_{V \in \mathcal{V}} \exists_{U \in \mathcal{U}} \forall_{x, x^{\prime} \in X}\left(\left(x, x^{\prime}\right) \in U \Rightarrow\left(f(x), f\left(x^{\prime}\right) \in V\right)\right.
$$

([9] Theorem 3.3.9). Uniformly continuous mappings between uniform spaces are strongly continuous and preserve total boundedness ([9], Propositions 3.3.2 and 3.3.7).

In the remainder of this paper we shall assume that our pre-apartness spaces are symmetric: if $A \bowtie B$, then $B \bowtie A$. Uniform apartness spaces are symmetric.

\section{PRECOMPACT SPACES}

We approach precompactness by reminding the reader of two theorems, the first of which is a consequence of Theorem (1.4) in Chapter 5 of [7].

Theorem 2.1. If $X$ is a compact - that is, inhabited, complete, and totally boundedmetric space, then there exists a uniformly continuous, open 5 mapping of $2^{\mathbf{N}}$ onto $X$.

The metric-space case of the second was given by Ishihara and Schuster [11] the proof of the following general case can be found in [9] (Theorem 3.3.18).

Theorem 2.2. Let $X$ be a totally bounded uniform space with a countable base of entourages. Then every strongly continuous mapping from $X$ into a uniform space is uniformly continuous.

In particular, every strongly continuous map from Baire space $2^{\mathbf{N}}$ into a uniform space is uniformly continuous.

With these two theorems in mind, we define an apartness space $X$ to be precompact if there exists a strongly continuous mapping from a dense subset of $2^{\mathbf{N}}$ onto a dense subset of $X 6$ An apartness space with a dense, precompact subset is itself precompact. The image of a precompact apartness space under a strongly continuous mapping is precompact.

Proposition 2.3. A precompact uniform space is totally bounded.

Proof. Let $X$ be a precompact uniform space. Then there exist a dense subset of $2^{\mathbf{N}}$ and a strongly continuous mapping $h$ of $D$ onto a dense subset of $X$. By Theorem $2.2, h$ is uniformly continuous. It follows (see earlier) that $h(D)$ is totally bounded; since $h(D)$ is dense in $X$, we see that $X$ itself is totally bounded.

Corollary 2.4. Let $f$ be a strongly continuous mapping of a precompact space $X$ into $\mathbf{R}$. Then $f(X)$ is totally bounded, and $\sup f, \inf f$ exist.

Proof. By Proposition 2.3, $f(X)$ is a totally bounded subset of $\mathbf{R}$. The rest of the proposition now follows from [8] (Proposition 2.2.5).

\footnotetext{
${ }^{5}$ Recall that an open mapping is one that preserves the openness of sets. In fact, the theorem in [7] proves more: there exists a uniform quotient map, a stronger notion than that of a uniformly continuous, open map, from $2^{\mathbf{N}}$ onto $X$.

${ }^{6}$ In classical topology, the name precompact is applied sometimes to sets that are dense a compact space, and sometimes to totally bounded sets in a uniform space. It is the latter application that we have in mind in making our current definition.

It is tempting to define precompactness in terms of images of arbitrary totally bounded uniform spaces under strongly continuous mappings. However, in the classical theory of proximity spaces, of which apartness spaces are the constructive counterpart, every proximity space satisfying the Efremovič property (for which see later) would be precompact in that sense, since it would have a totally bounded uniform structure compatible with its proximity/apartness structure; see [13] (page 72, (12.3)).
} 
Proposition 2.5. A metric space is precompact if and only if it is totally bounded.

Proof. Let $X$ be a totally bounded metric space, and $\widehat{X}$ its completion. Since $X$ is dense in $\widehat{X}$, the latter is totally bounded; it is therefore compact. By Theorem 2.1. there exists a uniformly continuous - and hence strongly continuous - open mapping $h$ of $2^{\mathbf{N}}$ onto $\widehat{X}$. Since $X$ is dense in $\widehat{X}$, the openness of $h$ ensures that $h^{-1}(X)$ is dense in $2^{\mathbf{N}}$. Hence $X$ is precompact.

The converse is a special case of Proposition 2.3.

We now digress to consider Cauchy nets and the extension of functions defined on uniform subspace: 7 of apartness spaces.

Let $X$ be a pre-apartness space. We shall need the the nested neighbourhoods property,

(NN) If $x \in-U$, then there exists $V \subset X$ such that $x \in-V$ and $\neg V \bowtie U$, and the property of weak symmetric separatedness,

(WSS) If $S \bowtie T$, then for each $x \in X$ there exists $U \subset X$ such that $x \in-U$ and $\neg(S-U \neq \varnothing \wedge T-U \neq \varnothing)$.

Uniform spaces possess both of these properties.

A net $s \equiv\left(x_{n}\right)_{n \in \mathfrak{D}}$ in $X$ is said to be totally Cauchy if for all subsets $A, B$ of $\mathfrak{D}$ with $s(A) \bowtie s(B)$, there exists $N \in \mathfrak{D}$ such that

$$
\neg\left(\exists_{m \in A}(m \succcurlyeq N) \wedge \neg \exists_{n \in B}(n \succcurlyeq N)\right),
$$

where, as is the case throughout this paper, $\succcurlyeq$ is the preorder relation on the index set $\mathfrak{D}$ of the net. Proposition 3.5.1 of [9] says that the property WSS is equivalent to every convergent net in $X$ being totally Cauchy. Proposition 3.5.15 of the same book tells us that if $X$ has the property $\mathbf{N N}$, and $s$ is a totally Cauchy net in $X$ that has a subnet converging to a limit $x \in X$, then $s$ itself converges to $x$; and Theorem 3.5.12 that if $(X, \mathcal{U})$ is a uniform (apartness) space, then every totally Cauchy net $\left(x_{n}\right)_{n \in \mathfrak{D}}$ in $X$ with a countable subnet is uniformly Cauchy, in the sense that

$$
\forall_{U \in \mathcal{U}} \exists_{N \in \mathfrak{D}} \forall_{m, n \succcurlyeq N}\left(\left(x_{m}, x_{n}\right) \in U\right) .
$$

Lemma 2.6. Let $(Z, \bowtie)$ be an apartness space, let $z \in Z$ have a countable base $\left(V_{n}\right)_{n \geq 1}$ of neighbourhoods in the apartness topology, and let $\left(z_{n}\right)_{n \in \mathfrak{D}}$ be a net in $Z$ that converges to $z$. Then there is a countable subnet $\left(z_{n_{k}}\right)_{k \geq 1}$ of $\left(z_{n}\right)_{n \in \mathfrak{D}}$ that converges to $z$.

Proof. There exists $n_{1} \in \mathfrak{D}$ such that $\left(z, z_{n}\right) \in V_{1}$ for all $n \succcurlyeq n_{1}$. Having constructed $n_{k} \in \mathfrak{D}$, pick $\nu \succcurlyeq n_{k}$ such that $\left(z, z_{n}\right) \in V_{k+1}$ for all $n \succcurlyeq \nu$. There exists $n_{k+1} \in \mathfrak{D}$ such that $n_{k+1} \succcurlyeq \nu$ and $n_{k+1} \succcurlyeq n_{k}$. Then $\left(z, z_{n}\right) \in V_{k+1}$ for all $n \succcurlyeq n_{k+1}$. This completes the inductive construction of a countable subnet $\left(z_{n_{k}}\right)_{k \geq 1}$ such that $\left(z, z_{n_{j}}\right) \in V_{k}$ whenever $j \succcurlyeq k$. Since $\left(V_{n}\right)_{n \geq 1}$ is a base of neighbourhoods of $z$, it follows that $\left(z_{n_{k}}\right)_{k \geq 1}$ converges to $z$.

\footnotetext{
${ }^{7}$ When we refer to a uniform (resp., metric) subspace $X$ of a pre-apartness space $Y$, we mean that there is a uniform structure $\mathcal{U}$ (resp., metric $\rho$ ) on $X$ such that the pre-apartness induced on $X$ by that on $Y$ coincides with the pre-apartness induced by $\mathcal{U}$ (resp., $\rho$ ).
} 
An apartness space is said to be first countable if each of its elements has a countable base of neighbourhoods in the apartness topology. First countability is used in several of the succeeding results, in order to enable us to work with sequences rather than general nets.

Lemma 2.7. Let $X$ be a dense uniform subspace of a first-countable, weakly symmetrically separated apartness space $Y$, and let $\widehat{X}$ be a complete uniform space in which $X$ is dense. Then there exists a mapping $g: Y \rightarrow \widehat{X}$ such that

(i) $g(x)=x$ for each $x \in X$, and

(ii) for each net $\left(x_{n}\right)_{n \in \mathfrak{D}}$ of elements of $X$ converging to $y \in Y$, the image net $\left(g\left(x_{n}\right)\right)_{n \in \mathfrak{D}}$ converges to $g(y)$.

Proof. Let $y \in Y$, and construct a net $\left(x_{n}\right)_{n \in \mathfrak{D}}$ in $X$ that converges to $y$ in the apartness topology. Since $Y$ has the property WSS, $\left(x_{n}\right)_{n \in \mathfrak{D}}$ is a totally Cauchy net in $X$. By Lemma 2.6, there is a countable subnet $\left(x_{n_{k}}\right)_{k \geq 1}$ of $\left(x_{n}\right)_{n \in \mathfrak{D}}$ that converges to $y$. It follows from a remark before Lemma 2.6 that the net $\left(x_{n}\right)_{n \in \mathfrak{D}}$ is uniformly Cauchy in $X$. Since $\widehat{X}$ is a complete uniform space, this net therefore converges to a unique limit $x_{\infty} \in \widehat{X}$. If $\left(x_{m}^{\prime}\right)_{m \in \mathfrak{M}}$ is another net in $X$ that converges to $y$ in the apartness topology, then it, too, has a countable subnet $\left(x_{m_{j}}^{\prime}\right)_{j \geq 1}$ and converges to a unique limit $x_{\infty}^{\prime}$ in $\widehat{X}$. Now, the sequences $\left(x_{n_{k}}\right)_{k \geq 1}$ and $\left(x_{m_{j}}^{\prime}\right)_{j \geq 1}$ both converge to $y$ in the space $Y$. Hence the sequence $s \equiv\left(x_{n_{1}}, x_{m_{1}}^{\prime} x_{n_{2}}, x_{m_{2}}^{\prime}, \ldots\right)$ converges to $y$. Since $y$ has the property WSS, this sequence is totally Cauchy in $X$; but $X$ is a uniform space, so, being itself a countable net, the sequence is uniformly Cauchy (again see the last remark before Lemma 2.6). It therefore converges to a unique limit in $\widehat{X}$. But it has a subsequence converging to $x_{\infty}$ and a subsequence converging to $x_{\infty}^{\prime}$. Since $\widehat{X}$, being a uniform space, has the property $\mathbf{N N}$, another remark before Lemma 2.6 shows that $s$ converges to both $x_{\infty}$ and $x_{\infty}^{\prime}$. By the uniqueness of limits in a uniform space, $x_{\infty}^{\prime}=x_{\infty}$. It now follows that setting $g(y)=x_{\infty}$ gives us a good definition of a function $g: Y \rightarrow \widehat{X}$ with properties (i) and (ii).

An extremely important property applicable to a pre-apartness space $X$ is the Efremovič property (a form of topological normality):

(EF) $S \bowtie T \Rightarrow \exists_{E \subset X}(A \bowtie \neg E \wedge E \bowtie B)$.

This property implies that

$$
\forall_{A, B \subset X}(A \bowtie B \Leftrightarrow \neg \neg A \bowtie B),
$$

and that

$$
\forall_{A, B \subset X}(A \bowtie B \Leftrightarrow \bar{A} \bowtie \bar{B})
$$

(9], Propositions 3.1.10, 3.1.7, 3.1.9, and Corollary 3.1.19).

Lemma 2.8. Let $(Y, \bowtie)$ be a first-countable, weakly symmetrically separated apartness space with the Efremovič property, let $X$ be a uniform subspace of $Y$, and let $\widehat{X}$ be a complete uniform space in which $X$ is dense. Then there exists a strongly continuous mapping $g$ of $Y$ onto a dense subset of $\widehat{X}$, such that $g(x)=x$ for each $x \in X$.

Proof. Construct the mapping $g$ as in Lemma 2.7. Given $y \in Y$, define

$$
\mathfrak{D}_{y} \equiv\{(x, U): x \in X, y \in-U, x \in-U\}
$$


and define the preorder $\succcurlyeq$ on $\mathfrak{D}_{y}$ by

$$
(x, U) \succcurlyeq\left(x^{\prime}, U^{\prime}\right) \Leftrightarrow-U \subset-U^{\prime} .
$$

Then $(x, U) \rightsquigarrow x$ is a net in $X$ that converges to $y$ in the space $Y$. We denote this net by $\left(x_{n}\right)_{n \in \mathfrak{D}_{y}}$; so if $n=(x, U) \in \mathfrak{D}_{y}$, then $x_{n}=x$. Note that, by the definition of the mapping $g$, this net converges to $g(y)$ in the space $\widehat{X}$.

The proof that $g$ is strongly continuous uses an argument very similar to one in the proof of Theorem 3.5.22 of [9]; we omit the details. Finally, since $g(Y)$ contains $X$, it is dense in $\widehat{X}$.

We are now able to give a limited generalisation of the theorem that a dense subspace of a totally bounded uniform space is totally bounded.

Proposition 2.9. Let $Y$ be a first-countable, weakly symmetrically separated, precompact apartness space with the Efremovič property, let $X$ be a dense uniform subspace of $Y$, and let $\widehat{X}$ be a complete uniform space in which $X$ is dense. Then $X$ is totally bounded.

Proof. By Lemma 2.8, there exists a strongly continuous mapping $g$ of $Y$ onto a dense subspace $Z$ of $\widehat{X}$ such that $g(x)=x$ for each $x \in X$. Then $g(Y)$, and therefore $Z$, is precompact; whence, by Proposition $2.3, Z$ is totally bounded. It follows that $X$, being clearly dense in $Z$, is totally bounded.

Corollary 2.10. Let $Y$ be a first-countable, weakly symmetrically separated, precompact apartness space with the Efremovič property, and $X$ a dense metric subspace of $Y$. Then $X$ is totally bounded and precompact.

Proof. In Proposition 2.9, take $\widehat{X}$ to be the metric completion of $X$, to show that $X$ is totally bounded. Reference to Proposition 2.5 shows that $X$ is precompact.

\section{UNIONS AND PRODUCTS OF PRECOMPACT SPACES}

Bearing in mind what happens with total boundedness in metric spaces, we would hope that precompactness passes to finite unions and products of apartness subspaces. To deal with unions, we need a construction that glues two apartness spaces together to make a new apartness space.

Let $X, Y$ be (symmetric) pre-apartness spaces, and let

$$
X \cup Y \equiv(X \times\{0\}) \cup(Y \times\{1\}),
$$

with equality and inequality those induced by their standard counterparts on $X \times Y$. Subsets of $X \cup Y$ have the form

$$
A \equiv\left(A^{0} \times\{0\}\right) \cup\left(A^{1} \times\{1\}\right)
$$

with $A^{0} \subset X$ and $A^{1} \subset Y$. We prove that the relation $\bowtie$ defined for subsets $A, B$ of $X \cup Y$ by

$$
A \bowtie_{X \uplus Y} B \Leftrightarrow\left(A^{0} \bowtie_{X} B^{0} \wedge A^{1} \bowtie_{Y} B^{1}\right)
$$

is an apartness, where such notations as $\bowtie_{X}$ have the obvious interpretation.

Clearly, $\bowtie_{X \uplus Y}$ is symmetric and satisfies (B1). Before verifying (B2), we note that for any $A \subset X \cup Y$ and for $i \in\{1,2\}$,

$$
(-A)^{i}=-A^{i} \text { and }(\sim A)^{i}=\sim A^{i} .
$$


For example,

$$
\begin{aligned}
(x, 0) \in-A & \Leftrightarrow(x, 0) \bowtie_{X \uplus Y} A \\
& \Leftrightarrow\{(x, 0)\}^{0} \bowtie A^{0} \text { and }\{(x, 0)\}^{1} \bowtie A^{1} \\
& \Leftrightarrow x \bowtie A^{0} \text { and } \varnothing \bowtie A^{1} \\
& \Leftrightarrow x \in-A^{0} .
\end{aligned}
$$

Thus if $\{(x, 0)\} \bowtie_{X \uplus Y} A$ in $X \cup Y$, then, by (B2) in $X, x \in \sim A^{0}$ and therefore $(x, 0) \in$ $\sim\left(A^{0} \times\{0\}\right)$. Since, clearly, $(x, 0) \in \sim\left(A^{1} \times\{1\}\right)$, we conclude that $(x, 0) \in \sim A$. Likewise, if $(y, 1) \bowtie_{X \uplus Y} A$ in $X \uplus Y$, then $(y, 1) \in \sim A$. This completes the proof that (B2) is satisfied.

For (B3), let $A_{1}, A_{2} \subset X$ and $B_{1}, B_{2} \subset Y$. Then

$$
\begin{aligned}
\left(A_{1} \cup A_{2}\right) \bowtie_{X \uplus Y}\left(B_{1} \cup B_{2}\right) & \Leftrightarrow \forall_{i \in\{0,1\}}\left(\left(A_{1} \cup A_{2}\right)^{i} \bowtie\left(B_{1} \cup B_{2}\right)^{i}\right) \\
& \Leftrightarrow \forall_{i \in\{0,1\}}\left(A_{1}^{i} \cup A_{2}^{i} \bowtie B_{1}^{i} \cup B_{2}^{i}\right) \\
& \Leftrightarrow \forall_{i \in\{0,1\}} \forall_{j, k \in\{1,2\}}\left(A_{j}^{i} \bowtie B_{k}^{i}\right) \\
& \Leftrightarrow \forall_{j, k \in\{1,2\}} \forall_{i \in\{0,1\}}\left(A_{j}^{i} \bowtie B_{k}^{i}\right) \\
& \Leftrightarrow \forall_{j, k \in\{1,2\}}\left(A_{j} \bowtie_{X \uplus Y} B_{k}\right) .
\end{aligned}
$$

To handle (B4), let $-A \subset \sim B$ in $X \cup Y$. Then $-A^{i}=(-A)^{i} \subset(\sim B)^{i}=\sim B^{i}$. Applying (B4) in $X$ and $Y$, we obtain $-A^{i} \subset-B^{i}$ and therefore $(-A)^{i} \subset(-B)^{i}$. Hence $-A \subset-B$ in $X \cup Y$.

Finally, consider $(x, 0) \in-A$ in $X \cup Y$. By the foregoing, $x \in-A^{0}$; whence, by (B5) in $X$, there exists $S^{0} \subset X$ such that $x \in-S^{0}$ and $X=-A^{0} \cup S^{0}$. Taking $S^{1}=Y$, we see that

and

$$
\{(x, 0)\}^{0}=\{x\} \bowtie_{X \uplus Y} S^{0} \times\{0\}
$$

Hence $x \in-S$, where

$$
\{(x, 0)\}^{1}=\varnothing \bowtie_{X \uplus Y} S^{1} \times\{1\} .
$$

$$
S \equiv\left(S^{0} \times\{0\}\right) \cup\left(S^{1} \times\{1\}\right) .
$$

Moreover, if $\left(x^{\prime}, 0\right) \in X \cup Y$, then $x^{\prime} \in-A^{0} \cup S^{0}=(-A)^{0} \cup S^{0}$; so either $\left(x^{\prime}, 0\right) \in-A$ or $\left(x^{\prime}, 0\right) \in S^{0} \times\{0\}$. On the other hand, if $(y, 1) \in X \cup Y$, then

$$
(y, 1) \in Y \times\{1\}=S^{1} \times\{1\} \subset S .
$$

Thus $X \cup Y=-A \cup\left(S^{0} \times\{0\}\right)$. A similar argument, starting with $(y, 1) \in-A$ in $X \cup Y$, completes the verification of (B5) and hence the proof that $\bowtie_{X \uplus Y}$ is an apartness on $X \cup Y$.

We call the space $X \cup Y$, taken with the apartness we have just constructed, the disjoint union of the apartness spaces $X$ and $Y$ (in that order).

Lemma 3.1. Let $X, Y$ be subspaces of an apartness space $E$. Then the mapping $g$ of $X \cup Y$ onto $X \cup Y$ defined by

$$
\begin{aligned}
& g(x, 0) \equiv x \quad(x \in X), \\
& g(y, 1) \equiv y \quad(y \in Y)
\end{aligned}
$$

is strongly continuous. 
Proof. Let $A \bowtie B$ in the subspace $X \cup Y$ of $E$. Then

$$
\left(g^{-1}(A)\right)^{0}=(A \cap X) \bowtie_{E}(B \cap X)=\left(g^{-1}(B)\right)^{0}
$$

and similarly $\left(g^{-1}(A)\right)^{1} \bowtie_{E}\left(g^{-1}(B)\right)^{1}$. Hence $g^{-1}(A) \bowtie_{X \uplus Y} g^{-1}(B)$.

The foregoing construction and lemma enable us to prove

Proposition 3.2. The union of two precompact subsets of an apartness space is precompact.

Proof. Let $X_{0}, X_{1}$ be precompact subsets of an apartness space $E$. Then for each $k$, there exist a dense subset $D_{k}$ of $2^{\mathrm{N}}$ and a strongly continuous mapping $h_{k}$ of $D_{k}$ onto a dense subset of $X_{k}$. Define a mapping

$$
w:\left(D_{1} \times\{0\}\right) \cup\left(D_{2} \times\{1\}\right) \rightarrow X_{0} \uplus X_{1}
$$

by

We first prove that

$$
w(\alpha, 0) \equiv\left(h_{1}(\alpha), 0\right) \text { and } w(\alpha, 1) \equiv\left(h_{2}(\alpha), 1\right)
$$

$$
S \equiv\left(h_{1}\left(D_{1}\right) \times\{0\}\right) \cup\left(h_{2}\left(D_{2}\right) \times\{1\}\right),
$$

the range of $w$, is dense in the apartness space $X_{0} \in X_{1}$. If $x \in X_{0}$ and $(x, 0) \in-A$ in $X_{0}$ ש $X_{1}$, then (as we showed on page 7) $x \in-A^{0}$ in $X_{0}$. Since $h_{1}\left(D_{1}\right)$ is dense in $X_{0}$, there exists $\alpha \in D_{1}$ such that $h_{1}(\alpha) \in-A^{0}$; since $\left\{\left(h_{1}(\alpha), 0\right)\right\}^{1}=\varnothing \bowtie A^{1}$ in $X_{1}$, we now see that $\left\{\left(h_{1}(\alpha), 0\right)\right\} \bowtie_{X_{0} \cup X_{1}} A$ and hence that $\left(h_{1}(\alpha), 0\right) \in-A$. Thus $h_{1}\left(D_{1}\right) \times\{0\}$ is dense in $X_{0} \times\{0\}$ relative to the apartness topology on $X_{0} \cup X_{1}$. Likewise, $h_{2}\left(D_{2}\right) \times\{1\}$ is dense in $X_{1} \times\{1\}$. Hence $S$ is dense in $X_{0} \cup X_{1}$.

To prove that $w$ is strongly continuous, let $A \bowtie_{X_{0} \uplus X_{1}} B$. Then $A^{0} \bowtie B^{0}$ in $X_{0}$, so $h_{1}^{-1}\left(A^{0}\right) \bowtie h_{1}^{-1}\left(B^{0}\right)$ in $D_{1}$. The definition of the product apartness on $2^{\mathbf{N}} \times\{0,1\}$ (see Section 3.7 of [9]) now yields

$$
w^{-1}\left(A^{0} \times\{0\}\right)=h_{1}^{-1}\left(A^{0}\right) \times\{0\} \bowtie h_{1}^{-1}\left(B^{0}\right) \times\{0\}=w^{-1}\left(B^{0} \times\{0\}\right) .
$$

On the other hand, denoting the $k$ th projection of the Cartesian product of two sets by $\mathrm{pr}_{k}$, we have

$$
\begin{aligned}
& w^{-1}\left(A^{0} \times\{0\}\right) \subset 2^{\mathbf{N}} \times\{0\}, \\
& w^{-1}\left(B^{1} \times\{1\}\right) \subset 2^{\mathbf{N}} \times\{1\}, \text { and } \\
& \operatorname{pr}_{2}\left(2^{\mathbf{N}} \times\{0\}\right) \bowtie \operatorname{pr}_{2}\left(2^{\mathbf{N}} \times\{1\}\right) ;
\end{aligned}
$$

whence, by definition of the product apartness on $2^{\mathbf{N}} \times\{0,1\}, w^{-1}\left(A^{0} \times\{0\}\right) \bowtie w^{-1}\left(B^{1} \times\right.$ $\{1\})$. Thus, by (B3) in the metric space $2^{\mathbf{N}} \times\{0,1\}$,

$$
\begin{aligned}
w^{-1}\left(A^{0} \times\{0\}\right) & \bowtie w^{-1}\left(B^{0} \times\{0\}\right) \cup w^{-1}\left(B^{1} \times\{1\}\right) \\
& =w^{-1}\left(\left(B^{0} \times\{0\}\right) \cup\left(B^{1} \times\{1\}\right)\right)=w^{-1}(B) .
\end{aligned}
$$

Similarly, $w^{-1}\left(A^{1} \times\{1\}\right) \bowtie w^{-1}(B)$; whence, by another application of (B3) in $2^{\mathbf{N}} \times\{0,1\}$, we obtain $w^{-1}(A) \bowtie w^{-1}(B)$. Thus $w$ is strongly continuous. Now, $2^{\mathbf{N}} \times\{0,1\}$, being the product of two compact metric spaces, is a compact metric space; so, by Theorem 2.1, there exists a uniformly - and hence strongly - continuous open mapping $f$ of $2^{\mathbf{N}}$ onto $2^{\mathbf{N}} \times\{0,1\}$. Since $\left(D_{1} \times\{0\}\right) \cup\left(D_{2} \times\{1\}\right)$ is dense in $2^{\mathbf{N}} \times\{0,1\}$ and $f$ is an open mapping,

$$
D \equiv f^{-1}\left(\left(D_{1} \times\{0\}\right) \cup\left(D_{2} \times\{1\}\right)\right)
$$


is dense in $2^{\mathbf{N}}$. Moreover, the restriction of $w \circ f$ to $D$ is a strongly continuous mapping of $D$ onto the dense subset $S$ of $X_{0} \uplus X_{1}$. On the other hand, the mapping $g$ of $X_{0} \uplus X_{1}$ onto $X_{0} \cup X_{1}$ defined in Lemma 3.1 is strongly continuous. Hence $g \circ w \circ f$ is a strongly continuous mapping of the dense subset $D$ of $2^{\mathbf{N}}$ onto the dense subset $h_{1}\left(D_{1}\right) \cup h_{2}\left(D_{2}\right)$ of $X_{0} \cup X_{1}$. Thus $X_{0} \cup X_{1}$ is precompact.

We now pass from unions to products.

Proposition 3.3. The product of two apartness spaces is precompact if and only if each of the factors is precompact.

Proof. Let $X_{0}, X_{1}$ be apartness spaces, and $X$ their product apartness space. If $X$ is precompact, then, since the projection mappings from $X$ onto its factors are strongly continuous ([9], Corollary 3.7.3) and hence preserve precompactness, we see that $X_{0}, X_{1}$ are precompact.

Suppose, conversely, that the two factor spaces are precompact. For each $k$, there exist a dense subset $D_{k}$ of $2^{\mathbf{N}}$ and a strongly continuous mapping $h_{k}$ of $D_{k}$ onto a dense subset of $X_{k}$. Then, by Proposition 3.7.6 of [9],

$$
\left(h_{1}, h_{2}\right):\left(\alpha_{1}, \alpha_{2}\right) \rightsquigarrow\left(h_{1}\left(\alpha_{1}\right), h_{2}\left(\alpha_{2}\right)\right)
$$

is a strongly continuous mapping of the dense subspace $D_{1} \times D_{2}$ of $2^{\mathbf{N}} \times 2^{\mathbf{N}}$ onto the dense subspace $h_{1}\left(D_{1}\right) \times h_{2}\left(D_{2}\right)$ of $X_{0} \times X_{1}$, which is therefore precompact.

This completes our introductory look at a possible generalisation of total boundedness in the context of an arbitrary apartness space. While most of the preceding results are exactly what one might wish for, there remain at least two undesirable features of the theory:

- the lack of a general analogue of the proposition that if a uniform space is totally bounded, then so are its dense subsets (Proposition 2.9 being a very restricted version of such an analogue);

- the inability to replace metric space by separable uniform space in Proposition 2.5. Moreover, since $2^{\mathbf{N}}$ is separable, so is each precompact space. Nevertheless, precompactness may well be worthy of further constructive exploration.

Acknowledgement. This work was done in part when the author was a guest of Professor Helmut Schwichtenberg in the Logic Section of the Mathematisches Institut, LudwigMaximilians-Universität, München.

\section{REFERENCES}

[1] P. Aczel and M. Rathjen: Notes on Constructive Set Theory, Report No. 40, Institut Mittag-Leffler, Royal Swedish Academy of Sciences, 2001.

[2] P. Aczel and M. Rathjen: Constructive Set Theory, in preparation.

[3] M.J. Beeson: Foundations of Constructive Mathematics, Springer Verlag, Heidelberg, 1985.

[4] E.A. Bishop: Foundations of Constructive Analysis, McGraw-Hill, New York, 1967.

[5] E.A. Bishop and D.S. Bridges: Constructive Analysis, Grundlehren der Math. Wissenschaften 279, Springer Verlag, Heidelberg-Berlin-New York, 1985.

[6] D.S. Bridges: 'Compactness notions for apartness spaces', Archiv for Math. Logic, online first, 2012; doi 10.1007/s00153-012-0279-6.

[7] D.S. Bridges and F. Richman: Varieties of Constructive Mathematics, London Math. Soc. Lecture Notes 97, Cambridge Univ. Press, 1987. 
[8] D.S. Bridges and L.S. Vîţă: Techniques of Constructive Analysis, Universitext, Springer, New York, 2006.

[9] D.S. Bridges and L.S. Vîţă: Apartness and Uniformity: A Constructive Development, in CiE series Theory and Applications of Computability, Springer Verlag, Heidelberg, 2011.

[10] H. Diener: 'Generalising compactness', Math. Logic Quart. 51(1), 49-57, 2008.

[11] H. Ishihara and P.M. Schuster: 'A constructive uniform continuity theorem', Quart. J. Math. 53, 185193, 2002.

[12] P. Martin-Löf: 'An intuitionistic theory of types', in Twenty-five Years of Constructive Type Theory (G. Sambin, J. Smith, eds), 127-172, Oxford Logic Guides 36, Clarendon Press, Oxford, 1998.

[13] S.A. Naimpally and B.D. Warrack: Proximity Spaces, Cambridge Tracts in Math. and Math. Phys. 59, Cambridge Univ. Press, Cambridge, U.K., 1970.

[14] T.A. Steinke: Constructive Notions of Compactness in Apartness Spaces, M.Sc. thesis, University of Canterbury, Christchurch, New Zealand, 2011.

[15] A.S. Troelstra and D. van Dalen: Constructivism in Mathematics: An Introduction (two volumes), North-Holland Publ. Co., Amsterdam, The Netherlands, 1988. 